\title{
DCF Throughput Analysis of IEEE 802.11a/g/n-based Mobile LAN over Correlated Fading Channel
}

\author{
Ha Cheol Lee \\ Dept. of Information and Telecom. Eng. Yuhan University \\ 185-34 Goean-Dong, Sosa-Gu, Bucheon City, Gyeonggi-Do, Korea \\ hclee@yuhan.ac.kr
}

\begin{abstract}
This paper explores a MAC (Medium Access Control) layer throughput with DCF (Distributed Coordination Function) protocol in the IEEE $802.11 \mathrm{a} / \mathrm{g} / \mathrm{n}$-based mobile LAN. It is evaluated in Rayleigh fading wireless channel, using theoretical analysis method. The DCF throughput performance is analyzed by using the number of stations with both variable payload size and mobile speed on the condition that fading margin and transmission probability are fixed. In the IEEE 802.11n, A-MSDU (MAC Service Data Unit Aggregation) scheme is considered and number of subframe is used as the variable parameter. It is identified that MAC efficiency of IEEE $802.11 \mathrm{n}$ is the best out of four schemes.
\end{abstract}

Keywords: Mobile LAN, MAC, Throughput, CSMA/CA, DCF, IEEE 802.11a/g/n.

\section{Introduction}

Over the past few years, mobile networks have emerged as a promising approach for future mobile IP applications. With limited frequency resources, designing an effective MAC protocol is a hot challenge. IEEE $802.11 \mathrm{~b} / \mathrm{g} / \mathrm{a} / \mathrm{n}$ networks are currently the most popular wireless LAN products on the market [1]-[3]. The conventional IEEE 802.11b and 802.11g/a specification provide up to 11 and $54 \mathrm{Mbps}$ data rates, respectively. However, the MAC protocol that they are based upon is the same and employs a CSMA/CA (Carrier Sense Multiple Access/Collision Avoidance) protocol with binary exponential back-off. IEEE 802.11 DCF is the de facto MAC protocol for wireless LAN because of its simplicity and robustness [1]. Therefore, considerable research efforts have been put on the investigation of the DCF performance over wireless LAN [4]-[7]. With the successful deployment of IEEE 802.11a/b/g wireless LAN and the increasing demand for real-time applications over wireless, the IEEE 802.11n Working Group standardized a new MAC and PHY (Physical) Layer specification to increase the bit rate to be up to $600 \mathrm{Mbps}$ [8],[9]. The throughput performance at the MAC layer can be improved by aggregating several frames before transmission [10]. Frame aggregation not only reduces the transmission time for preamble and frame headers, but also reduces the waiting time during CSMA/CA random backoff period for successive frame transmissions. The frame aggregation can be performed within different sub-layers. In $802.11 \mathrm{n}$, frame aggregation can be performed either by A-MPDU (MAC Protocol Data Unit Aggregation) or A-MSDU (MAC Service Data Unit Aggregation). Although frame aggregation can increase the throughput at the MAC layer under ideal channel conditions, a larger aggregated frame will cause each station to wait longer before its next chance for channel access. Under error-prone channels, corrupting a large aggregated frame may waste a long period of channel time and lead to a lower MAC efficiency [11]-[13]. On the other hand, wireless LAN mobile stations that are defined as the stations that access the LAN while in motion are considered in this paper [7]. The previous paper analyzed the IEEE $802.11 \mathrm{~b} / \mathrm{g} / \mathrm{n}$ MAC performance for wireless LAN with fixed stations, not for wireless LAN with mobile stations [4]-[7],[10]-[13]. On the contrary, reference 7 and 14 analyzed the MAC performance for IEEE 802.11 wireless LAN with mobile stations, but considered only IEEE 802.11 and 802.11g/a wireless LAN specification. So, this paper extends the previous researches and

Received: April $10^{\text {th }}, 2011$. Accepted: December $20^{\text {th }}, 2011$ 
analyzes the IEEE 802.11n MAC performance for wireless LAN with mobile stations. In other words, we will present the analytical evaluation of saturation throughput with bit errors appearing in the transmitting channel. IEEE 802.11 g/a/n PHY and MAC layer focused in this paper are reviewed and frame error rate of mobile wireless channel is derived in section 2 . The DCF saturation throughput is theoretically derived in section 3 and numerical results are analyzed in section 4. Finally, it is concluded with section 5.

\section{Wireless access architecture}

\section{A. 802.11a/g/n-based wireless access architecture}

Figure 1 shows ad-hoc mode operation in the mobile LAN. The protocols of the various layers are called the protocol stack. The TCP/IP protocol stack consists of five layers: the physical, data link, network, transport and application layers. 802.11 of Figure 1 means physical layer and data link layer which consists of MAC and LLC (Logical Link Control) sublayers. And this paper is focused on physical layer and MAC sublayer. An ad-hoc network might be formed when people with laptops get together and want to exchange data in the absence of a centralized AP (Access Point).

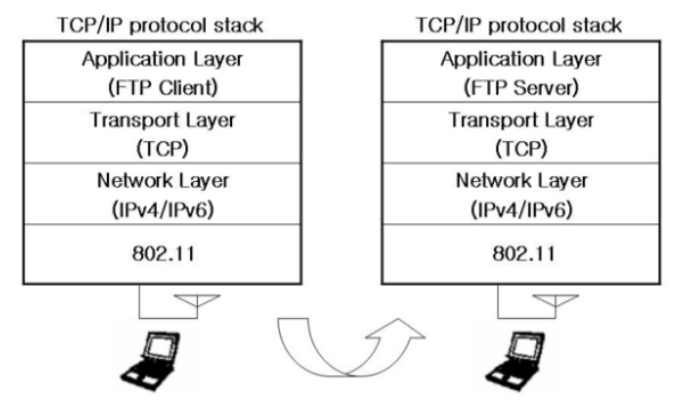

Figure 1. Ad-hoc mode operation in the mobile LAN

Table 1. Shows IEEE 802.11n OFDM parameter compared to IEEE 802.11a/g.

\begin{tabular}{|c|c|c|c|}
\hline \multirow{2}{*}{ Standards } & \multirow{2}{*}{ IEEE 802.11a/g } & \multicolumn{2}{|c|}{ IEEE 802.11n } \\
\hline & & Mandatory & Optional \\
\hline Maximum transmission rate (Mbps) & 54 & 130 & 600 \\
\hline Bandwidth(MHz) & 20 & 20 & 40 \\
\hline FFT size & 64 & 64 & 128 \\
\hline $\begin{array}{l}\text { Number of subcarrier } \\
(\text { data }+ \text { pilot })\end{array}$ & $\begin{array}{c}52 \\
(48+4)\end{array}$ & $\begin{array}{c}56 \\
(52+4)\end{array}$ & $\begin{array}{c}114 \\
(108+6)\end{array}$ \\
\hline Multi-antenna scheme & signal antenna & 2 Tx MIMO & $\begin{array}{c}\text { 3,4 Tx MIMO } \\
\text { Tx Beam forming } \\
\text { STBC }\end{array}$ \\
\hline Channel coding & $\begin{array}{c}\text { Convolutional code } \\
(1 / 2,2 / 3,3 / 4)\end{array}$ & $\begin{array}{c}\text { Convolutional code } \\
(1 / 2,2 / 3,3 / 4,5 / 6)\end{array}$ & $\begin{array}{c}\text { LDPC } \\
(1 / 2,2 / 3,3 / 4,5 / 6)\end{array}$ \\
\hline Modulation & \multicolumn{3}{|c|}{ BPSK, QPSK, 16-QAM, 64-QAM } \\
\hline Spatial stream & 1 & $1 \sim 2$ & $1 \sim 4$ \\
\hline Guard interval(ns) & 800 & 800 & 400 \\
\hline Subcarrier interval & $312.5 \mathrm{KHz}$ & $312.5 \mathrm{KHz}$ & $312.5 \mathrm{KHz}$ \\
\hline FFT period & $3.2 \mu \mathrm{s}$ & $3.2 \mu \mathrm{s}$ & $3.2 \mu \mathrm{s}$ \\
\hline Symbol period & $4 \mu \mathrm{s}$ & $4 \mu \mathrm{s}$ & $4 \mu \mathrm{s}$ \\
\hline
\end{tabular}




\section{B. 802.11a/g PHY/MAC layer}

Figure 2 shows the $802.11 \mathrm{a} / \mathrm{g}$-based physical and MAC layer protocol stack and typical frame structure focused in this paper. When a higher layer pushes a user packet down to the MAC layer as a MAC-SDU (MSDU), the M-HDR (MAC layer header) and FCS (trailer) are added before and after the MSDU, respectively and form a MPDU (MAC-PDU). The PHY layer is again divided into a PLCP (Physical Layer Convergence Protocol) sub-layer and a PMD (Physical Medium Dependent) sub-layer. Similarly the PLCP preamble and P-HDR (PLCP header) are attached to the MPDU at the PLCP sub-layer. Different IFS (Inter Frame Space)s are added depending on the type of MPDU.

IEEE 802.11a operates in the $5 \mathrm{GHz}$ band and uses OFDM (Orthogonal Frequency Division Multiplexing). The achievable data rates are 6, 9, 12, 18, 24, 36, 48, and $54 \mathrm{Mbps}$. IEEE $802.11 \mathrm{~g}$ uses DSSS, OFDM, or both at the $2.4 \mathrm{GHz}$ ISM band to provide high data rates of up to $54 \mathrm{Mbps}$. IEEE $802.11 \mathrm{~g}$ device can operate with an IEEE $802.11 \mathrm{~b}$ device. Combined use of both DSSS and OFDM is achieved through the provision of four different physical layers. The four different physical layers defined in the IEEE 802.11g standards are ERPDSSS/CCK, ERP-OFDM, ERP-DSSS/PBCC and DSSS-OFDM. The standards that support the highest data rate of $54 \mathrm{Mbps}$ are ERP-OFDM and DSSS-OFDM. ERP-OFDM is a new physical layer in IEEE $802.11 \mathrm{~g}$ and OFDM is used to provide IEEE $802.11 \mathrm{a}$ data rates at the $2.4 \mathrm{GHz}$ band. DSSS-OFDM is a new physical layer that uses a hybrid combination of DSSS and OFDM. The packet physical header is transmitted using DSSS, while the packet payload is transmitted using OFDM. Figure 3 shows basic access scheme of CSMA/CA mechanism. The SIFS (Short Inter-Frame Space) and the slot time are determined by the physical layer. DIFS (Distributed Inter-Frame Space) is defined based on the above two intervals.

\begin{tabular}{|c|c|c|c|c|c|c|c|}
\hline & M- HDR & MAC- SDU & FCS & & \\
\hline & & & \multicolumn{3}{|c|}{ MAC- PDU } & & \\
\hline & Preamble & P- HDR & \multicolumn{3}{|c|}{ PLCP-SDU } & & \\
\hline & \multicolumn{5}{|c|}{ PLCP- PDU } & & \\
\hline IFS & \multicolumn{5}{|c|}{ BitStream (PMD- SDU) } & IFS & BO \\
\hline
\end{tabular}

(a) Protocol stack of physical and MAC layer

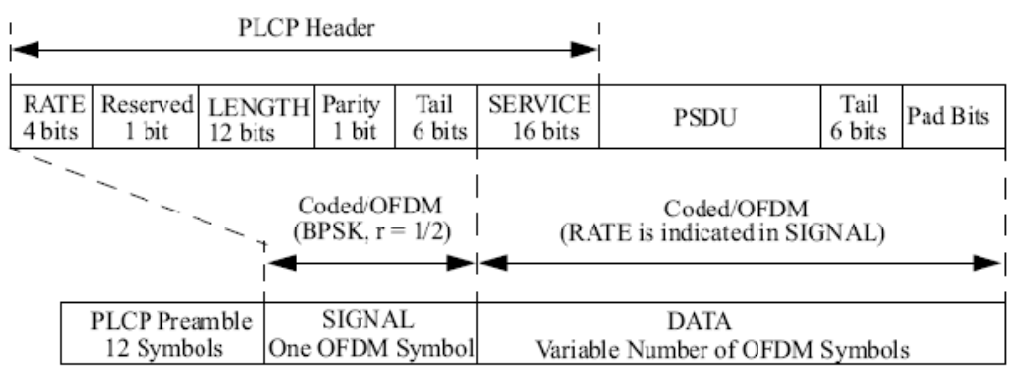

(b) $802.11 \mathrm{a}$ and $802.11 \mathrm{~g}$ ERP-OFDM frame

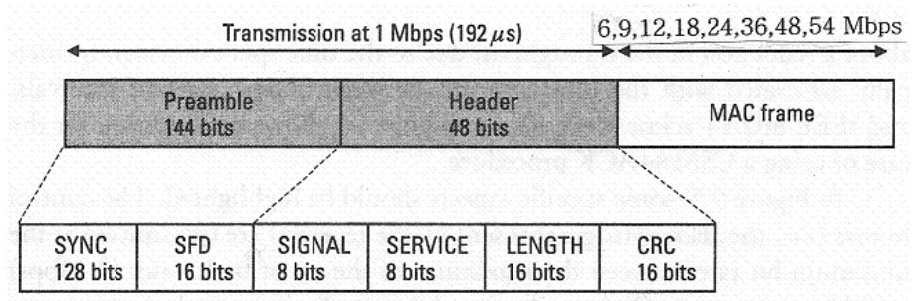

(c) $802.11 \mathrm{~g}$ DSSS-OFDM frame

Figure 2. Protocol stack and frame structure of IEEE $802.11 \mathrm{a} / \mathrm{g} /$-based mobile LAN 


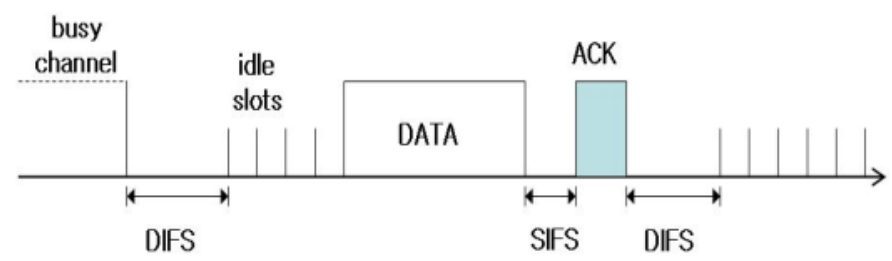

(a) basic access scheme

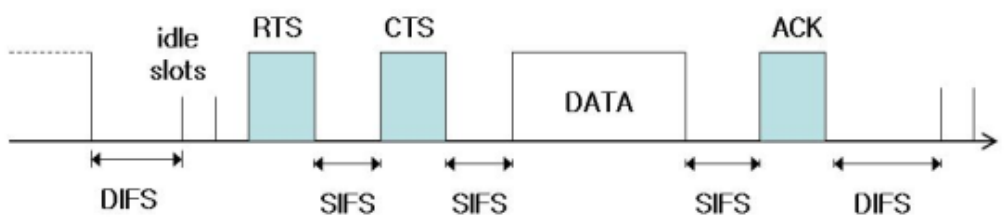

(b) RTS/CTS scheme

Figure 3. IEEE 802.11g/a DCF channel access mechanism

Table 2. Shows parameters of the IEEE 802.11a physical layer and table 3 shows parameters of the different IEEE $802.11 \mathrm{~g}$ physical layers

\begin{tabular}{|c|c|c|c|c|c|}
\hline $\begin{array}{c}\text { Data Rate } \\
(\text { Mbits/sec) }\end{array}$ & Modulation & $\begin{array}{c}\text { Coding } \\
\text { Rate } \\
(\mathbf{R})\end{array}$ & $\begin{array}{c}\text { Coding Bits } \\
\text { Per Subcarrier } \\
\left(\boldsymbol{N}_{\text {BPSC })}\right.\end{array}$ & $\begin{array}{c}\text { Coded Bits per } \\
\text { OFDM symbol } \\
\left(\boldsymbol{N}_{\text {CBPS }}\right)\end{array}$ & $\begin{array}{c}\text { Data Bits Per } \\
\text { OFDM Symbol } \\
\left(\boldsymbol{N}_{\text {DBPS }}\right)\end{array}$ \\
\hline 6 & BPSK & $1 / 2$ & 1 & 48 & 24 \\
\hline 9 & BPSK & $3 / 4$ & 1 & 48 & 36 \\
\hline 12 & QPSK & $1 / 2$ & 2 & 96 & 48 \\
\hline 18 & QPSK & $3 / 4$ & 2 & 96 & 72 \\
\hline 24 & $16-\mathrm{QAM}$ & $1 / 2$ & 4 & 192 & 96 \\
\hline 36 & $16-\mathrm{QAM}$ & $3 / 4$ & 4 & 192 & 144 \\
\hline 48 & 64-QAM & $2 / 3$ & 6 & 288 & 216 \\
\hline 54 & $64-\mathrm{QAM}$ & $3 / 4$ & 6 & 288 & 292 \\
\hline
\end{tabular}

Table 3. Parameters of the different IEEE $802.11 \mathrm{~g}$ physical layers

\begin{tabular}{|c|c|c|c|c|c|}
\hline \multirow{2}{*}{$\begin{array}{l}\text { Physical } \\
\text { layer }\end{array}$} & \multirow{2}{*}{$\begin{array}{l}\text { Supported } \\
\text { rates (Mb/s) }\end{array}$} & \multicolumn{2}{|c|}{$\begin{array}{l}\text { PLCP preamble } \\
\text { + header delay }\end{array}$} & \multicolumn{2}{|c|}{$\begin{array}{l}\text { PLCP preamble } \\
+ \text { header length }\end{array}$} \\
\hline & & Long & Short & Long & Short \\
\hline $\begin{array}{l}\text { ERP-DSSS } \\
\text { (mandatory) }\end{array}$ & $1,2,5.5,11$ & $192 \mu \mathrm{s}$ & $96 \mu \mathrm{s}$ & 192 bits & 120 bits \\
\hline $\begin{array}{l}\text { ERP-OFDM } \\
\text { (mandatory) }\end{array}$ & $\begin{array}{l}6,9,12,18, \\
24,36,48,54\end{array}$ & \multicolumn{2}{|c|}{$20 \mu \mathrm{s}$} & \multicolumn{2}{|c|}{40 bits $^{1}$} \\
\hline $\begin{array}{l}\text { ERP-PBCC } \\
\text { (optional) }\end{array}$ & $\begin{array}{l}1,2,5.5,11 \\
22,33\end{array}$ & $192 \mu \mathrm{s}$ & $96 \mu \mathrm{s}$ & 192 bits & 120 bits \\
\hline $\begin{array}{l}\text { DSSS-OFDM } \\
\text { (optional) }\end{array}$ & $\begin{array}{l}6,9,12,18 \\
24,36,48,54\end{array}$ & $192 \mu \mathrm{s}$ & $96 \mu \mathrm{s}$ & 192 bits & 120 bits \\
\hline
\end{tabular}




\section{802.11n PHY/MAC layer}

At the MAC layer, 802.11n use several new MAC, including the frame aggregation, block acknowledgement, and bi-directional data transmission. There are two ways to perform frame aggregation at the MAC layer as shown in Figure 4. The first technique is by concatenating
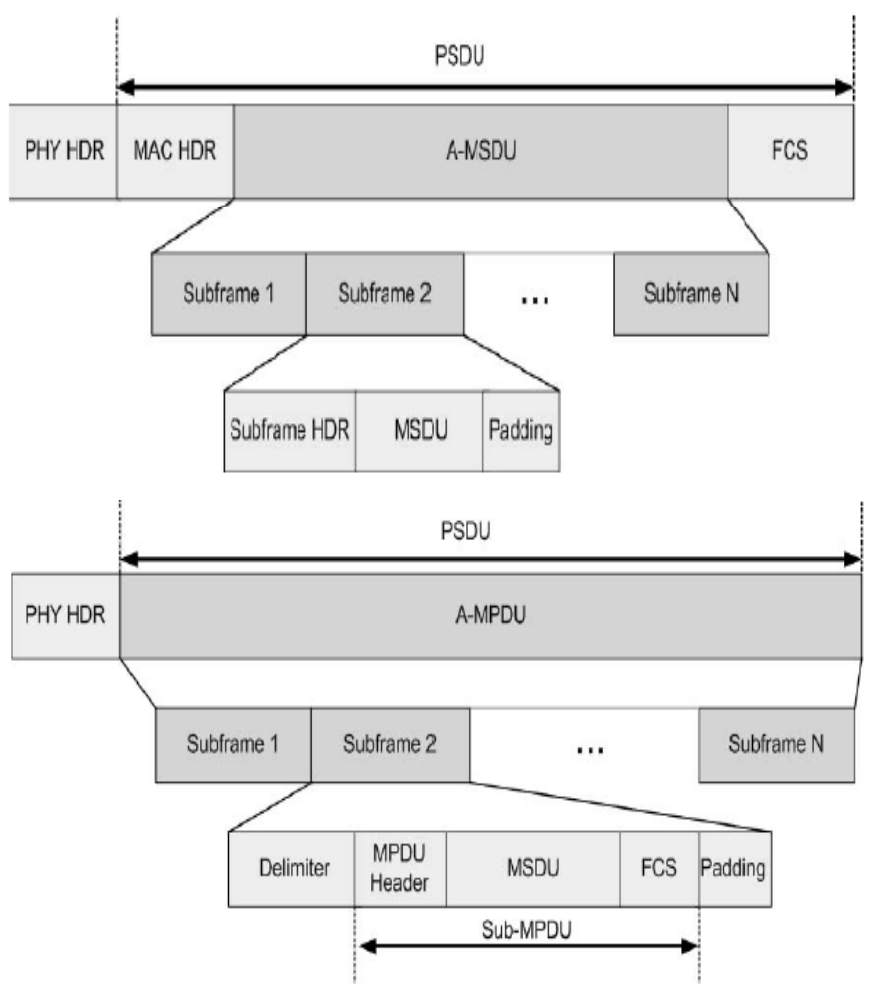

(a) $802.11 \mathrm{n}$ Frame Format for A-MSDU and A-MPDU

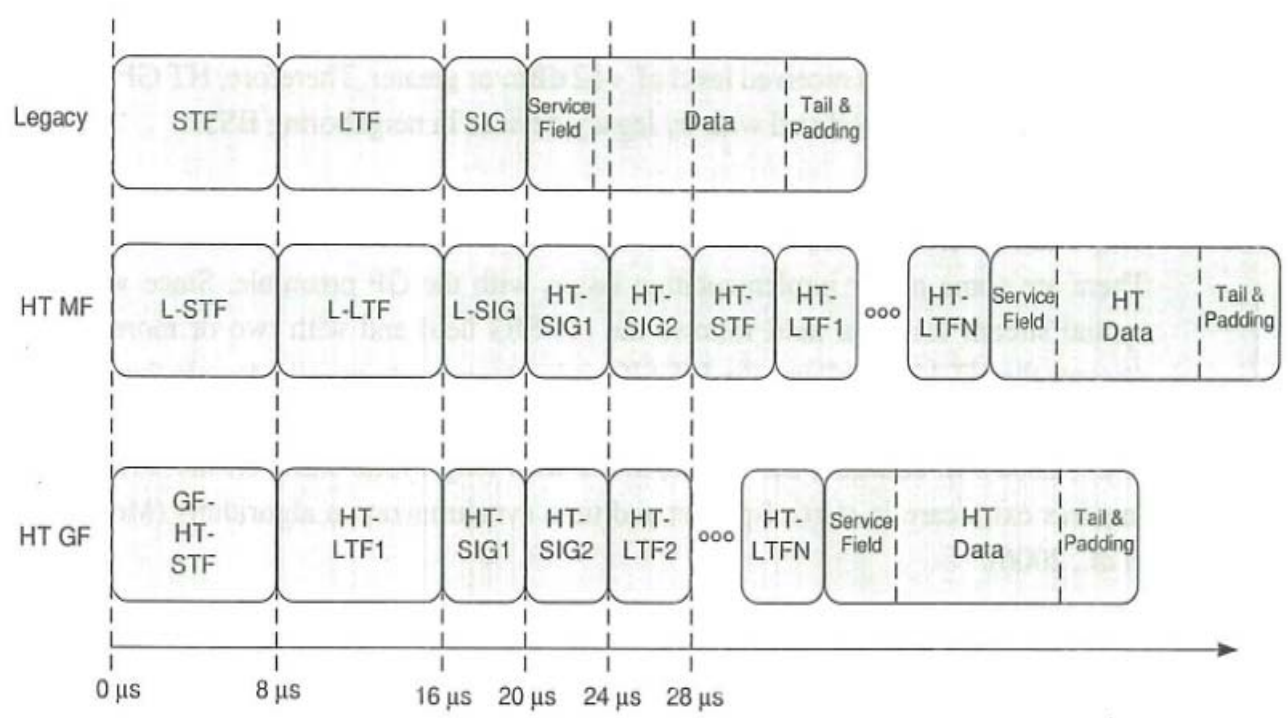

(b) Timing of the preamble fields in legacy, MF and GF

Figure 4. Frame structure of IEEE 802.11n-based mobile LAN 
several MSDUs (MAC Service Data Units) to form the data payload of a large MPDU (MAC Protocol Data Unit). The PHY header and MAC header, along with the FCS (Frame Check Sequence), are then appended to form the PSDU (Physical Service Data Unit). This technique is known as A-MSDU. The second technique is called A-MPDU. It begins with each MSDU appending with its own MAC header and FCS to form a sub-MPDU. An MPDU delimiter is then inserted before each sub-MPDU. Padding bits are also inserted so that each sub-MPDU is a multiple of 4 bytes in length, which can facilitate subframe delineation at the receiver. Then, all the sub-MPDUs are concatenated to form a large PSDU. Figure 4 also shows timing of the preamble fields in legacy, MF(Mixed Format) and GF (Green Field). At the PHY layer, 802.11n will use MIMO (Multiple-Input Multiple-Output) and OFDM. It supports up to a transmission rate of $600 \mathrm{Mbps}$ and is backward compatible with IEEE 802.11a/b/g. IEEE $802.11 \mathrm{n}$ provides support for both $2.4 \mathrm{GHz}$ and $5 \mathrm{GHz}$ frequency bands at the same time. IEEE 802.11n defines implicit and explicit TxBF (Transmit BeamForming) methods and STBC (Space-Time Block Coding), which improves link performance over MIMO with basic SDM (Spatial-Division Multiplexing). It also defines a new optional LDPC (Low Density Parity Check) encoding scheme, which provides better coding performance over the basic convolutional code.

The possible timing sequences for A-MPDU and A-MSDU in the uni-directional transfer case are shown in Figure 5. If RTS/CTS (Request To Send/Clear To Send) is used, the current transmission sequence of RTS-CTS-DATA (Data frame)-ACK (Acknowledgement) only allows the sender to transmit a single data frame. The DATA frame represents either an AMPDU or an A-MSDU frame. The system time can be broken down into virtual time slots where each slot is the time interval between two consecutive countdown of backoff timers by non-transmitting stations. The IEEE $802.11 \mathrm{n}$ also specifies a bi-directional data transfer method. In the bi-directional data transfer method, the receiver may request a reverse data transmission in the CTS control frame. The sender can then grant a certain medium time for the receiver on the reverse link. The transmission sequence will then become RTS-CTS-DATAfDATAr-ACK. This facilitates the transmission of some small feedback packets from the receiver and may also enhance the performance of TCP (Transmission Control Protocol) which requires the transmission of TCP ACK segments. BACK (Block Acknowledgement) can be used to replace the previous ACK frame. The BACK can use a bit map to efficiently acknowledge each individual sub-frame within the aggregated frame.

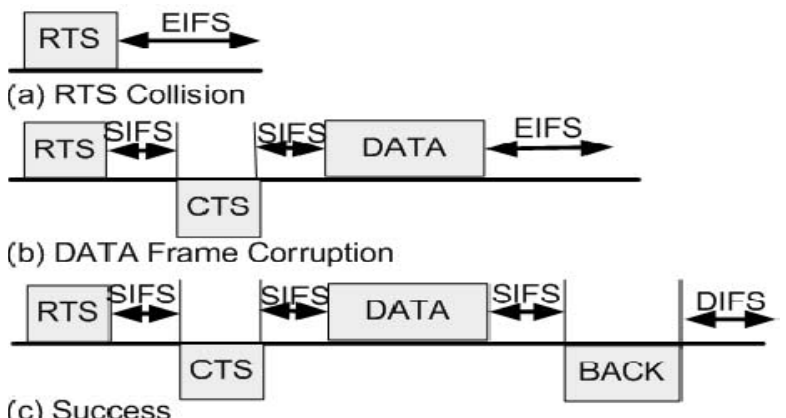

Figure 5. IEEE 802.11n Uni-directional RTS/CTS Access Scheme 
Table 4. Parameters of the IEEE 802.11n physical layer, MCS Rates 0-31

\begin{tabular}{|c|c|c|c|c|c|c|c|}
\hline \multirow{3}{*}{$\begin{array}{l}\text { MCS } \\
\text { Index }\end{array}$} & \multirow{3}{*}{ Modulation } & \multirow{3}{*}{$\begin{array}{l}\text { Coding } \\
\text { Rate }\end{array}$} & \multirow{3}{*}{$\begin{array}{l}\text { Spatial } \\
\text { Streams }\end{array}$} & \multicolumn{4}{|c|}{ 802.11n Data Rate (Mbps) } \\
\hline & & & & \multicolumn{2}{|c|}{ 20-MHz } & \multicolumn{2}{|c|}{ 40-MHz } \\
\hline & & & & L-GI & S-GI & L-GI & S-GI \\
\hline 0 & BPSK & $1 / 2$ & 1 & 6.5 & 7.2 & 13.5 & 15 \\
\hline 1 & QPSK & $1 / 2$ & 1 & 13 & 14.4 & 27 & 30 \\
\hline 2 & QPSK & $3 / 4$ & 1 & 19.5 & 21.7 & 40.5 & 45 \\
\hline 3 & 16-QAM & $1 / 2$ & 1 & 26 & 28.9 & 54 & 60 \\
\hline 4 & 16-QAM & $3 / 4$ & 1 & 39 & 43.3 & 81 & 90 \\
\hline 5 & 64-QAM & $2 / 3$ & 1 & 52 & 57.8 & 108 & 120 \\
\hline 6 & 64-QAM & $3 / 4$ & 1 & 58.5 & 65 & 122 & 135 \\
\hline 7 & 64-QAM & $5 / 6$ & 1 & 65 & 72.2 & 135 & 150 \\
\hline 8 & BPSK & $1 / 2$ & 2 & 13 & 14.4 & 27 & 30 \\
\hline 9 & QPSK & $1 / 2$ & 2 & 26 & 28.9 & 54 & 60 \\
\hline 10 & QPSK & $3 / 4$ & 2 & 39 & 43.3 & 81 & 90 \\
\hline 11 & 16-QAM & $1 / 2$ & 2 & 52 & 57.8 & 108 & 120 \\
\hline 12 & 16-QAM & $3 / 4$ & 2 & 78 & 86.7 & 162 & 180 \\
\hline 13 & 64-QAM & $2 / 3$ & 2 & 104 & 116 & 216 & 240 \\
\hline 14 & 64-QAM & $3 / 4$ & 2 & 117 & 130 & 243 & 270 \\
\hline 15 & 64-QAM & $5 / 6$ & 2 & 130 & 144 & 270 & 300 \\
\hline 16 & BPSK & $1 / 2$ & 3 & 19.5 & 21.7 & 40.5 & 45 \\
\hline 17 & QPSK & $1 / 2$ & 3 & 39 & 43.3 & 81 & 90 \\
\hline 18 & QPSK & $3 / 4$ & 3 & 58.5 & 65 & 121.5 & 135 \\
\hline 19 & 16-QAM & $1 / 2$ & 3 & 78 & 86.7 & 162 & 180 \\
\hline 20 & 16-QAM & $3 / 4$ & 3 & 117 & 130 & 243 & 270 \\
\hline 21 & 64-QAM & $2 / 3$ & 3 & 156 & 173.3 & 324 & 360 \\
\hline 22 & 64-QAM & $3 / 4$ & 3 & 175.5 & 195 & 364.5 & 405 \\
\hline 23 & 64-QAM & $5 / 6$ & 3 & 195 & 216.7 & 405 & 450 \\
\hline 24 & BPSK & $1 / 2$ & 4 & 26 & 28.9 & 54 & 60 \\
\hline 25 & QPSK & $1 / 2$ & 4 & 52 & 57.8 & 108 & 120 \\
\hline 26 & QPSK & $1 / 2$ & 4 & 78 & 86.7 & 162 & 180 \\
\hline 27 & 16-QAM & $1 / 2$ & 4 & 104 & 115.6 & 216 & 240 \\
\hline 28 & 16-QAM & $3 / 4$ & 4 & 156 & 173.3 & 324 & 360 \\
\hline 29 & 64-QAM & $2 / 3$ & 4 & 208 & 231.1 & 432 & 480 \\
\hline 30 & 64-QAM & $3 / 4$ & 4 & 234 & 260 & 486 & 540 \\
\hline 31 & 64-QAM & $5 / 6$ & 4 & 260 & 288.9 & 540 & 600 \\
\hline
\end{tabular}




\section{Frame error rate}

Mobile wireless channel is assumed to be flat fading Rayleigh channel with Jake spectrum. The channel is in fading states or inter-fading states by evaluating a certain threshold value of received signal power level. If and only if the whole frame is in inter-fading state, there is the successful frame transmission. If any part of frame is in fading duration, the frame is received in error. In the fading channel fading margin is considered and defined as $\rho=R_{\text {req }} / R_{r m s}$, Where $R_{\text {req }}$ is the required received power level and $R_{r m s}$ is the mean received power. Generally, the fading duration and inter-fading duration can be taken to be exponentially distributed for $\rho<-10 \mathrm{~dB}$. With the above assumptions, let Tpi be the frame duration, then the frame error rate is given by (1) [7].

$$
F E R=1-\frac{T i}{T i+T_{f}} P(t i>T p i)
$$

Where, $t_{i}$ is inter-fading duration and $t_{f}$ is fading duration. Ti is the mean value of the random variable $t_{i}$ and $T_{f}$ is the mean value of the random variable $t_{f} . P(t i>T p i)$ is the probability that inter-fading duration lasts longer than Tpi. Since exponential distribution is assumed for $t_{i}, P\left(t_{i}>T p i\right)=\exp \left(-\frac{T p i}{T i}\right)$. For Rayleigh fading channel, the average fading duration is given by (2).

$$
T i=\frac{\exp (\rho)-1}{f d \sqrt{2 \pi \rho}}
$$

$T_{i}+T_{f}$ is $\frac{1}{N_{f}}$, where $N_{f}$ is the level crossing rate, which is given by $f d \sqrt{2 \pi \rho} \exp (-\rho) . f_{d}$

is the maximum Doppler frequency and evaluated as $\frac{v}{\lambda} \cdot v$ is the mobile speed and $\lambda$ is wavelength. Frame error rate can be expressed by (3).

$$
F E R=1-\exp \left(-\rho-f_{d} \sqrt{2 \pi \rho} T p i\right)
$$

Equation (3) shows that frame error rate is determined by fading margin, maximum Doppler frequency and frame duration. Since fading margin and maximum Doppler frequency are hard to dynamically control, the only controllable parameter is frame duration to get required frame error rate. For the RTS/CTS access mode, the frame duration $T_{p i}$ is $T_{H}+T_{R T S}+T_{C T S}+T_{D A T A}+T_{A C K} . T_{H}$ is preamble transmission time + PLCP header transmission time + MAC header transmission time. TDATA is MSDU transmission time and TACK is ACK frame transmission time. $T_{R T S}$ is RTS frame transmission time and $T_{C T S}$ is CTS frame transmission time.

\section{DCF throughput analysis}

The back-off procedure of the DCF protocol is modeled as a discrete-time, two-dimensional Markov chain. Figure 6 shows the Bianchi's Markov chain model for the back-off window size [11]. We define $W=C W_{\min }$. Let $m$, the maximum back-off stage, be such value that $C W_{\max }=2^{m} W$. We also define $W_{i}=2^{i} W$, where $i \in(0, m)$ is called the back-off stage. Let $s(t)$ be the stochastic process representing the back-off stage $(0, \ldots, m)$ of the station at time $t$. $p$ is the probability that a transmission is collided or unsuccessfully executed. 


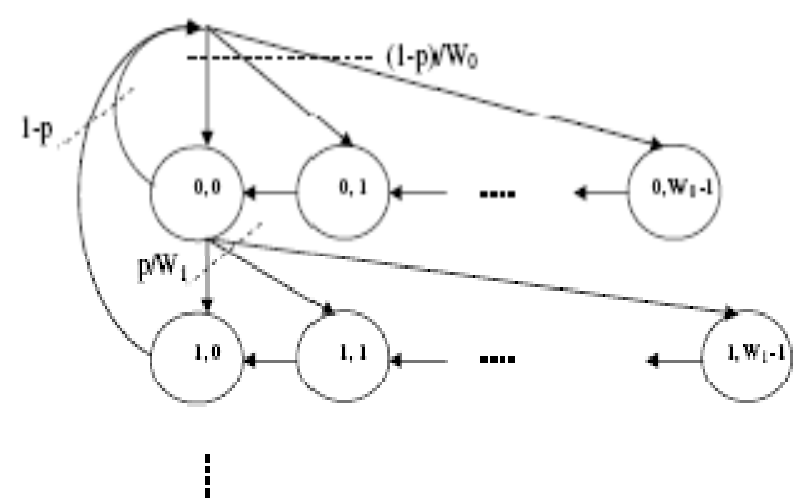

Figure 6. Markov chain model for the backoff window size

Table 5 shows physical and MAC layer parameters of IEEE $802.11 \mathrm{a} / \mathrm{g} / \mathrm{n}$-based mobile LAN.

\begin{tabular}{|c|c|}
\hline Parameter & Explanation \\
\hline FER & Frame error rate \\
\hline$\tau$ & Packet transmission probability \\
\hline$N$ & Number of stations \\
\hline$P$ & Payload size \\
\hline$T_{\text {RTS }}$ & RTS frame transmission time \\
\hline$T_{\text {CTS }}$ & CTS frame transmission time \\
\hline$T_{H}$ & $\begin{array}{l}\text { PLCP preamble transmission time }+ \text { PLCP header transmission time }+ \\
\text { MAC header transmission time }\end{array}$ \\
\hline$T_{\text {DATA }}$ & Payload transmission time \\
\hline$T_{A C K}$ & ACK frame transmission time \\
\hline$T_{B A C K}$ & Block ACK frame transmission time \\
\hline$\sigma$ & Slot time \\
\hline$T_{\text {SIFS }}$ & SIFS time \\
\hline$T_{\text {DIFS }}$ & DIFS time \\
\hline TEIFS & EIFS time \\
\hline$C W \min$ & Minimum backoff window size \\
\hline$C W \max$ & Maximum backoff window size \\
\hline
\end{tabular}

We will present the analytical evaluation of saturation throughput with bit errors appearing in the transmitting channel. The number of stations $\mathrm{n}$ is assumed to be fixed and each station always has packets for transmission. In other words, we operate in saturation conditions, the transmission queue of each station is assumed to be always nonempty.

Let $S$ be the normalized system throughput, defined as the fraction of time in which the channel is used to successfully transmit payload bits. $P_{t r}$ is the probability that there is at least one transmission in the considered slot time. Since $n$ stations contend on the channel and each transmits with probability $\tau$, we get

$P_{t r}$

$$
=1-(1-\tau)^{n}
$$




\section{A. 802.11a/g DCF throughput}

Saturation throughput is represented as shown in (5).

$$
\begin{aligned}
& S=\frac{P_{s} P_{t r} P}{\left(1-P_{t r}\right) \sigma+P_{t r} P_{s} T_{s}+P_{t r}\left(1-P_{s}\right) T_{c}} \\
& =\frac{n \tau(1-\tau)^{n-1}(1-F E R) P}{(1-\tau)^{n} \sigma+n \tau(1-\tau)^{n-1}(1-F E R) T_{S}+\left[1-(1-\tau)^{n}\right] T_{C}-n \tau(1-\tau)^{n-1}(1-F E R) T_{C}}
\end{aligned}
$$

$P_{S}$ is the probability that a transmission successfully occurs on the channel and is given by the probability that exactly one station transmits on the channel, conditioned on the fact that at least one station transmits.

$$
P_{s}=\frac{n \tau(1-\tau)^{n-1}(1-F E R \quad)}{P_{t r}}
$$

The average amount of payload information successfully transmitted in a slot time is $P_{t r} P_{s} P$ since a successful transmission occurs in a slot time with probability $P_{t r} P_{s}$. The average length of a slot time is readily obtained considering that, with probability $1-P_{t r}$, the channel is empty, with probability $P_{t r} P_{S}$ it contains a successful transmission, and with probability $P_{t r}\left(1-P_{S}\right)$ it contains a collision. Where $T_{S}$ is the average time the channel is sensed busy because of a successful transmission, and $T_{c}$ is the average time the channel is sensed busy by each station during a collision or error. $\sigma$ is the duration of an empty slot time. In the RTS/CTS access scheme, we obtain,

$$
\begin{aligned}
& T_{S}=T_{R T S}+T_{C T S}+T_{D A T A}+T_{\text {ACK }}+T_{\text {DIFS }}+3 T_{\text {SIFS }} \\
& T_{C}=T_{\text {RTS }}+T_{\text {EIFS }}=T_{\text {RTS }}+T_{\text {SIFS }}+T_{\text {ACK }}+T_{\text {DIFS }}
\end{aligned}
$$

\section{B. 802.11n DCF throughput}

In the uni-directional case shown in Fig. 5, the saturation throughput can be calculated as follows[12,13].

$$
\begin{aligned}
& S=\frac{E_{p}}{E_{t}}=\frac{L_{p} P_{t r} P_{s}\left(1-P_{e}\right)}{T_{\text {idle }} P_{\text {idle }}+T_{c} P_{\text {tr }}\left(1-P_{s}\right)+T_{e} P_{\text {err }}+T_{\text {succ }} P_{\text {succ }}} \\
& = \\
& \frac{L_{p} n \tau(1-\tau)^{n-1}\left(1-P_{e}\right)}{(1-\tau)^{n} \sigma+n \tau(1-\tau)^{n-1}\left(1-P_{e}\right) T_{\text {succ }}+\left[1-(1-\tau)^{n}-n \tau(1-\tau)^{n-1}\right] T_{c}+n \tau(1-\tau)^{n-1} P_{e} T_{e}}
\end{aligned}
$$


where $E_{p}$ is the number of payload information bits successfully transmitted in a virtual time slot, and $E_{t}$ is the expected length of a virtual time slot. $P_{e}$ is the error probability on condition that there is a successful RTS/CTS transmission in the time slot and can be defined as the FER (Frame Error Rate). $P_{\text {idle }}$ is the probability of an idle slot. $P_{s}$ is the probability for a non-collided transmission. $P_{e r r}$ is the transmission failure probability due to error (no collisions but having transmission errors). $P_{\text {succ }}$ is the probability for a successful transmission without collisions and

transmission errors. $T_{\text {idle }}, T_{c}$ and $T_{\text {succ }}$ are the idle, collision and successful virtual time slot's length. $T_{e}$ is the virtual time slot length for an error transmission sequence. $L_{p}$ is the aggregated frame's payload length. In the RTS/CTS scheme, we obtain,

$$
\begin{aligned}
& T_{C}=T_{\text {RTS }}+T_{\text {EIFS }} \\
& T_{\text {SUCC }}=T_{\text {RTS }}+T_{\text {CTS }}+T_{\text {DATA }}+T_{\text {BACK }}+3 T_{\text {SIFS }}+T_{\text {DIFS }} \\
& T_{e}=T_{\text {RTS }}+T_{\text {CTS }}+T_{\text {DATA }}+T_{\text {EIFS }}+2 T_{\text {SIFS }}
\end{aligned}
$$

\section{Numerical results}

This section evaluated DCF throughput of the IEEE 802.11a/g-based mobile LAN with the maximum physical transmission rate of $54 \mathrm{Mbps}$ and that of the IEEE 802.11n-based mobile LAN with the physical transmission rate of $130 \mathrm{Mbps}$ considering $20 \mathrm{MHz}$ MCS (modulation and coding scheme) parameters for two spatial streams, as shown in Figure 8. Out of the four different physical layers defined in the IEEE 802.11g standard, both ERP-OFDM and DSSSOFDM standard are only used owing to their maximum transmission rate of $54 \mathrm{Mbps}$ in this evaluation [14]. And the three common packets passed down to the MAC layer are 60 bytes (TCP ACK), 576 bytes (typical size for web browsing) and 1,500 bytes (the maximum size for Ethernet) in length. In the IEEE 802.11n-based mobile LAN, the number of packets aggregated in one MAC frame varies from 1 to 100 , which leads to an aggregated frame's payload length ( $L_{p}$ ) from 60, 576 and 1,500 bytes to 6, 57.6 and 150 Kbytes. In the Figure 7(a) Figure 7(c), the symbol fer $(\rho, v, P)$ shows frame error rate of IEEE $802.11 \mathrm{a} / \mathrm{g}$. In the Figure $7(\mathrm{~d})$, the symbol fer (ns, $\rho, v, \mathrm{P}$ ) shows frame error rate of IEEE 802.11n with the horizontal parameter of subframe' payload size. In the Figure 7(e), the symbol fer ( $\rho, n s, v, P$ ) shows frame error rate of IEEE $802.11 \mathrm{n}$ using the number of subframes as the horizontal parameter. It is generally identified that the higher mobile speed is, the higher fer is. In case of payload size, the same result mentioned above is also acquired. In the Figure $8(\mathrm{a}) \sim$ Figure $8(\mathrm{c})$, the symbol $\mathrm{S}(P, \rho$, $v, n, \tau)$ shows the saturation throughput over error-prone channel according to the number of stations $(n)$ for common packet sizes $(P)$ on the condition that packet transmission probability $(\tau)$, mobile velocity $(v)$ and fading margin $(\rho)$ are fixed. In the Figure $8(\mathrm{~d})$ and Figure 8(e), the symbol S ( $n s, P, \rho, v, n, \tau)$ and $\mathrm{S}(\mathrm{P}, n s, \rho, v, n, \tau)$ respectively shows the saturation throughput over error-prone channel according to the number of stations $(n)$ and the typical number of packets aggregated in one MAC frame ( $n s$ ) for two subframe length on the condition that packet transmission probability $(\tau)$, mobile velocity $(v)$ and fading $\operatorname{margin}(\rho)$ are fixed. For example, in the Figure 8(a), if the number of stations is 7, packet transmission probability is 0.05 , packet length is 1,500 and fading margin is 0.01 , mobile station with the speed of $1.25 \mathrm{~m} / \mathrm{s}$ can get the throughput of $27.238 \mathrm{Mbps}$, whereas mobile station with the speed of $25 \mathrm{~m} / \mathrm{s}$ can get the throughput of $26.968 \mathrm{Mbps}$. In the Figure 8(d), if 
subframe length is 30 and the same conditions mentioned above are applied, mobile station with the speed of $1.25 \mathrm{~m} / \mathrm{s}$ can get the throughput of $113.511 \mathrm{Mbps}$ with six stations, whereas mobile station with the speed of $25 \mathrm{~m} / \mathrm{s}$ can get the throughput of $84.607 \mathrm{Mbps}$. Also, Figure $8(\mathrm{a} \sim \mathrm{d})$ shows that the longer frame (or subframe) length is, the higher throughput is. And, for the same frame (or subframe) length, the higher speed is, the lower throughput is. As the results of evaluation, we also know that there is optimum number of stations to maximize saturation throughput under the error-prone channel. Specially, in Figure 8(e), the number of subframes is considered and it is identified that there is optimum number of subframes to maximize saturation throughput under the error-prone channel.

In conclusion, we obtained the fact that there exist an optimal number of stations (or subframes) to maximize the saturation throughput under the error-prone channel. Also, we can identify that the larger payload (or subpayload) size be, the higher saturation throughput be. And if a mobile velocity of station is increased, the throughput is decreased a little. Out of the three different physical layers defined in this analysis with the maximum transmission rate of $54 \mathrm{Mbps}$, which are IEEE 802.11g ERP-OFDM, IEEE 802.11g DSSS-OFDM and IEEE 802.11a OFDM, The DCF saturation throughput of IEEE 802.11a OFDM is the highest at all the channel environments. In the case of IEEE 802.11n, because A-MSDU (MAC Service Data Unit Aggregation) scheme is applied, it is identified that MAC efficiency of IEEE $802.11 \mathrm{n}$ is the best out of all four schemes.

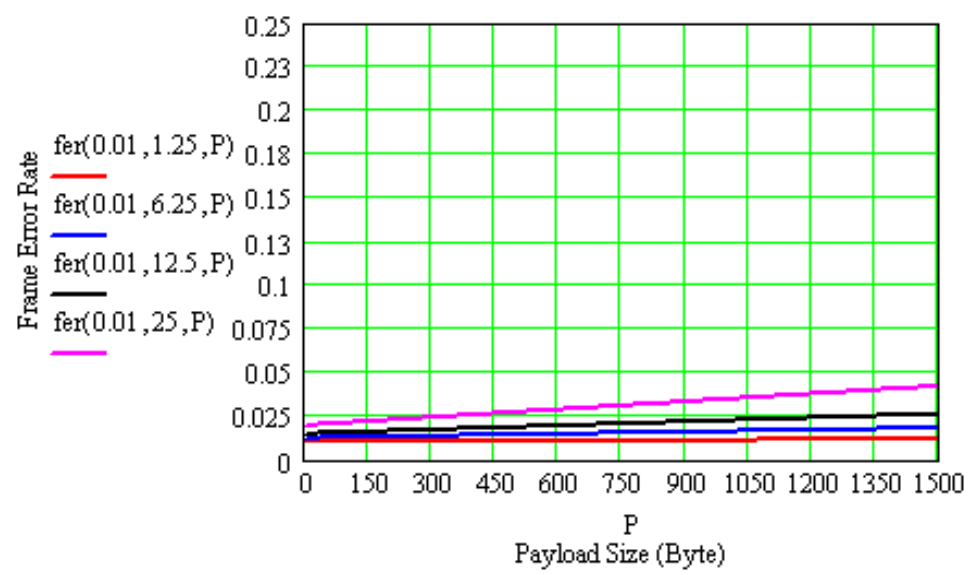

(a) 802.11a OFDM (54 Mbps)

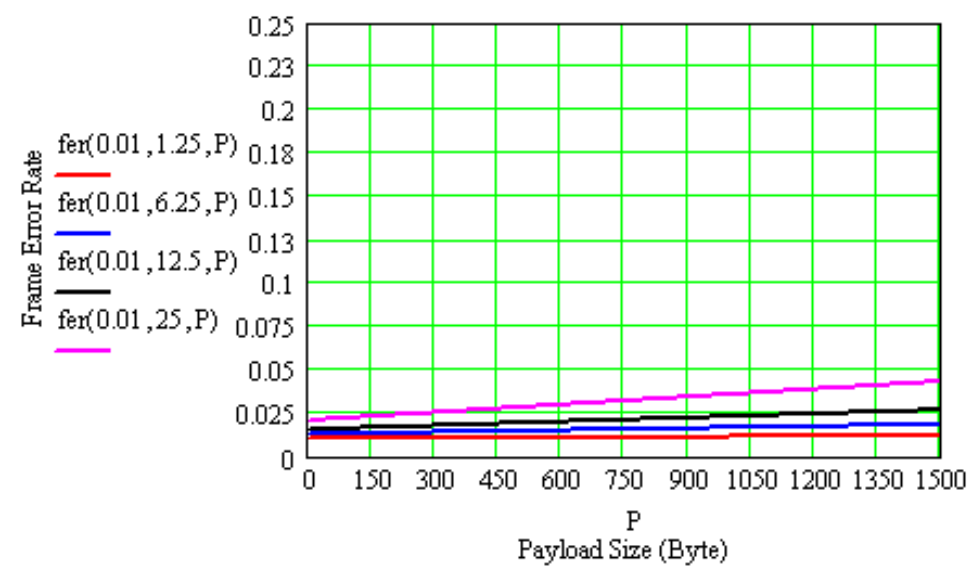

(b) $802.11 \mathrm{~g}$ ERP-OFDM (54 Mbps) 

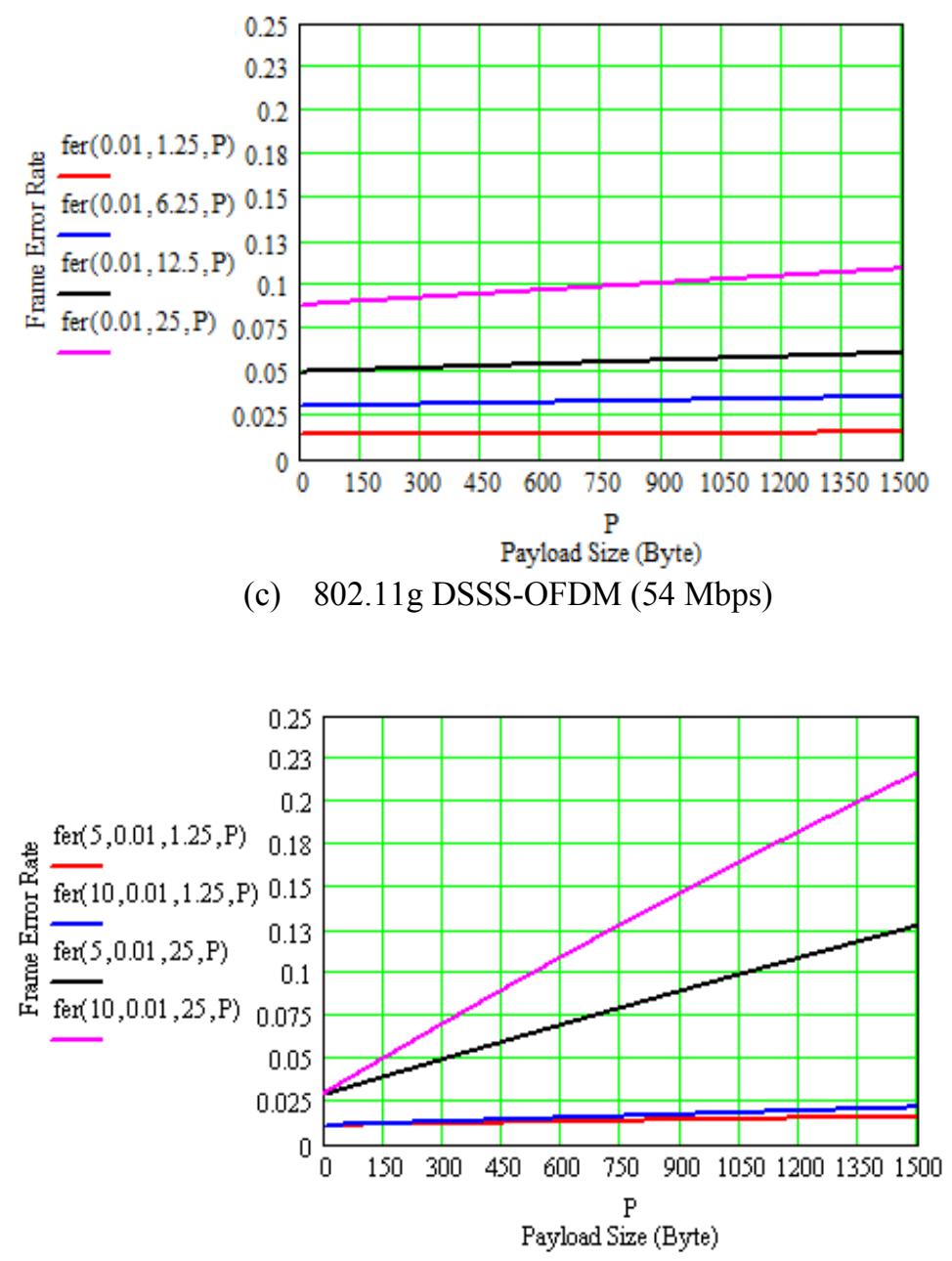

(d) $802.11 \mathrm{n}$ OFDM (58.5 Mbps, Payload size)

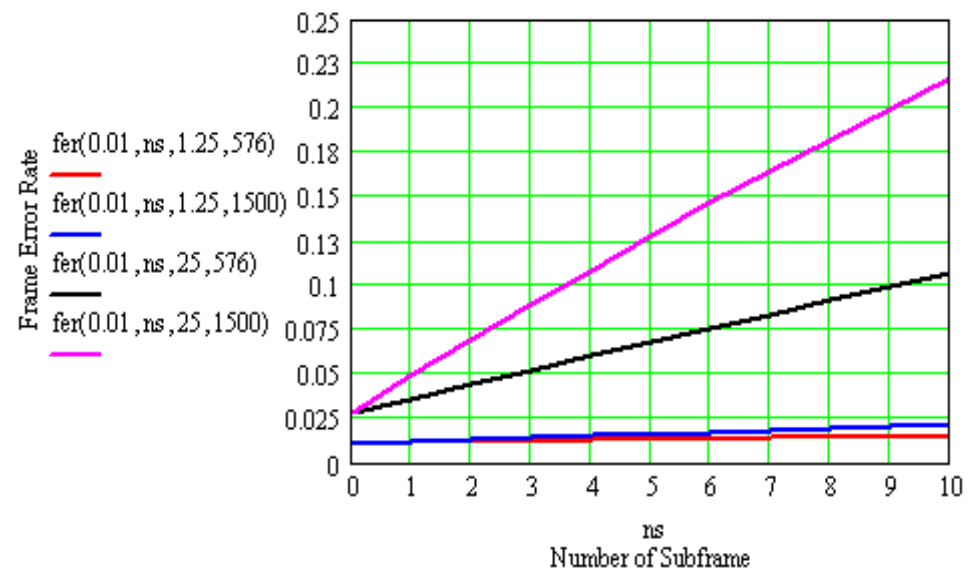

(e) $802.11 \mathrm{n}$ OFDM (58.5 Mbps, number of subframe)

Figure 7. Frame error rate of IEEE $802.11 \mathrm{a} / \mathrm{g} / \mathrm{n}$ mobile LAN 


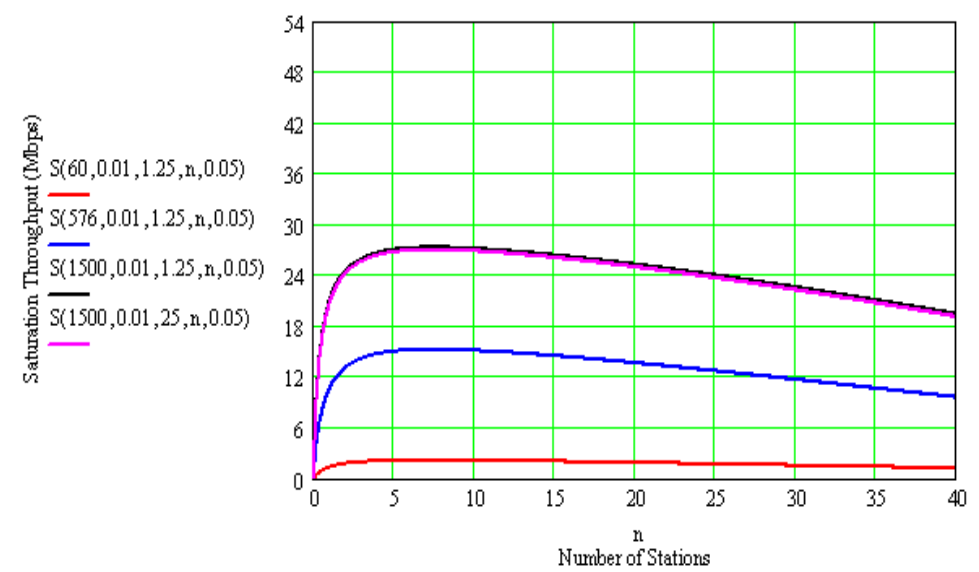

(a) $802.11 \mathrm{a}$ OFDM (54 Mbps)

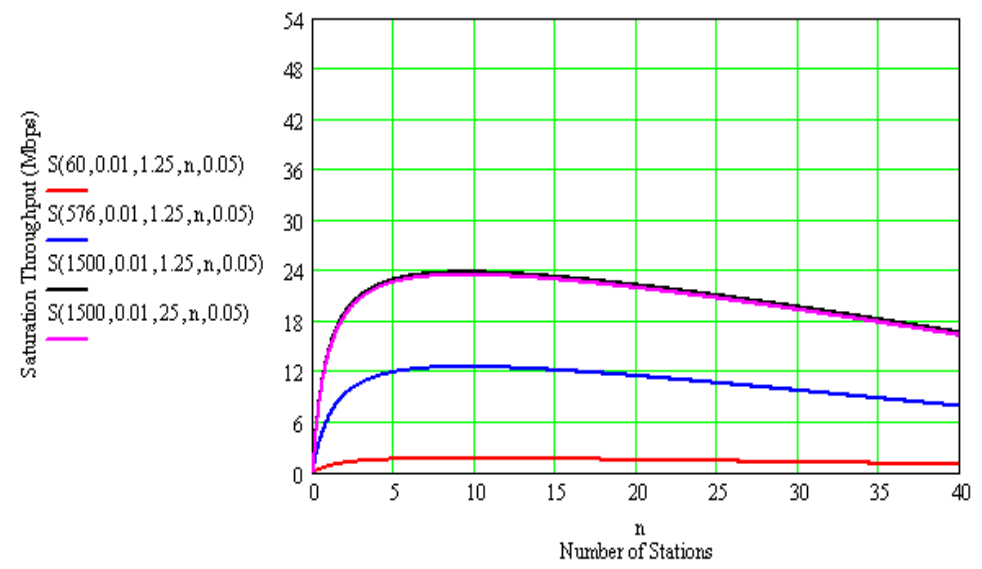

(b) $802.11 \mathrm{~g}$ ERP-OFDM (54 Mbps)

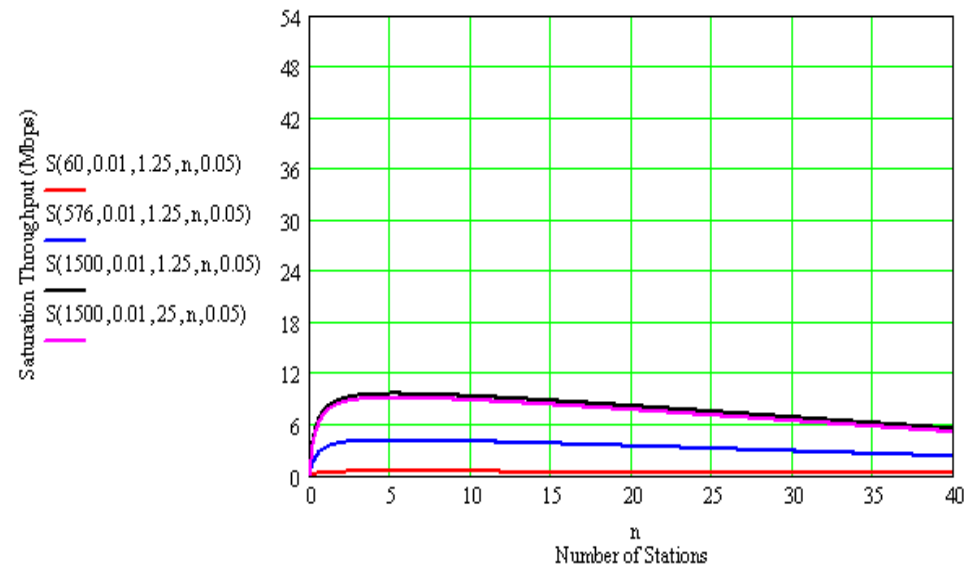

(c) $802.11 \mathrm{~g}$ DSSS-OFDM (54 Mbps) 


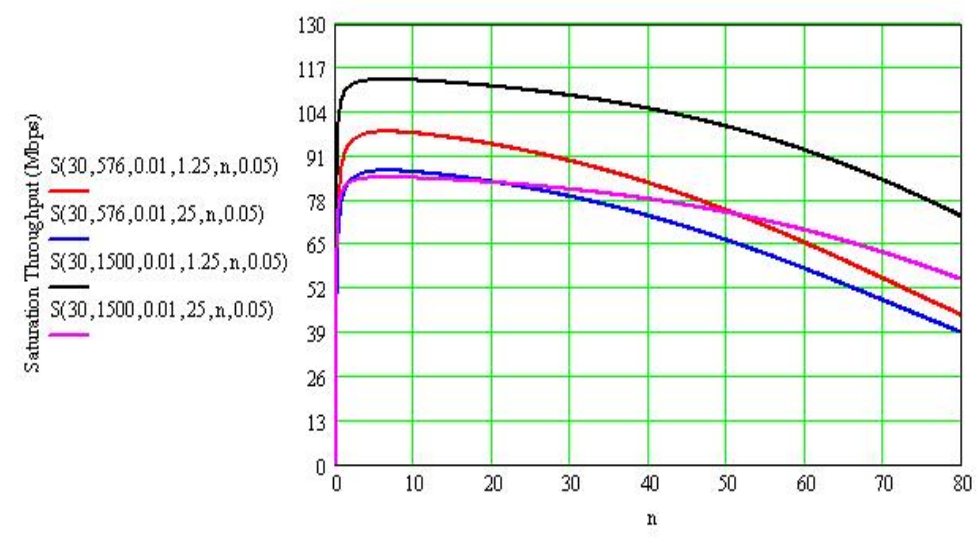

Number of Stations

(d) $802.11 \mathrm{n}$ OFDM (130 Mbps, number of stations)

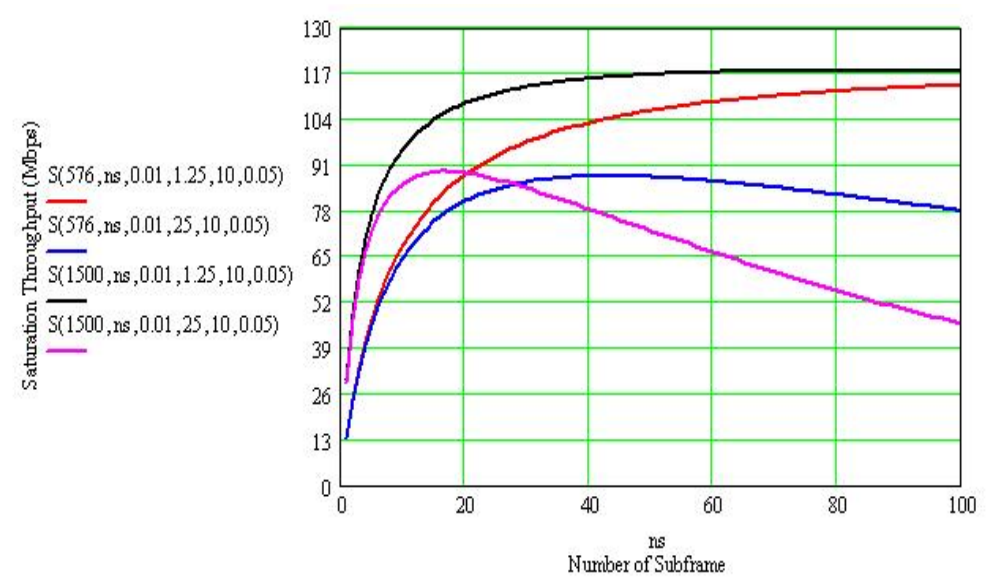

(e) $802.11 \mathrm{n}$ OFDM (130 Mbps, number of subframe)

Figure 8. DCF throughput of IEEE $802.11 \mathrm{a} / \mathrm{g} / \mathrm{n}$ mobile LAN

\section{Conclusions}

This paper explored the saturation throughput performance of DCF protocol in the IEEE $802.11 \mathrm{a} / \mathrm{g} / \mathrm{n}$-based mobile LAN under the error-prone channel. IEEE 802.11a and IEEE 802.11g have the same maximum transmission rate of $54 \mathrm{Mbps}$, but the DCF saturation throughput of IEEE $802.11 \mathrm{a}$ is higher than that of IEEE $802.11 \mathrm{~g}$. Of the two $802.11 \mathrm{~g}$ standards, DCF saturation throughput of IEEE $802.11 \mathrm{~g}$ ERP-OFDM is higher than that of IEEE 802.11g DSSS-OFDM. We are recognizing that a IEEE 802.11n-based device can operate with a IEEE 802.11 legacy devices, but IEEE 802.11a-based device does not operate with a IEEE $802.11 \mathrm{~b} / \mathrm{g}$-based device. So this interoperability have to be considered for designing and constructing IEEE $802.11 \mathrm{a} / \mathrm{g} / \mathrm{n}$-based mobile LAN.

\section{References}

[1] Upkar Varshney, "The Status and Future of 802.11-based Wireless LANs," IEEE Computer, Jun. 2003, pp. 102-105.

[2] C. Siva Ram Murthy and B. S. Manoj, “Ad Hoc Wireless Networks," Prentice Hall, 2004, pp. 172-179. 
[3] Carlos de Morais Cordeiro and Dharma Prakash Agrawal, "Ad Hoc \& Sensor Networks," World Scientific, 2006, pp. 172-187.

[4] Zuoyin Tang, Zongkai Yang, Jianhua He and Yanwei Liu, "Impact of Bit Errors on the Performance of DCF for Wireless LAN," IEEE, pp. 529-533, 2002.

[5] Dimitris Vassis, George Kormentzas, Angelos Rouskas and llias Maglogiannis, "The IEEE 802.11g Standard for High data rate WLANs," IEEE Network, pp. 21-26, May/June, 2005.

[6] Nghia T. Dao and Robert A. Malaney, "Throughput Performance of Saturated 802.11g Networks," AusWireless 2007, 2007.

[7] Xi Yong, Wei Ji Bo and Zhuang Zhao Wen, "Throughput Analysis of IEEE 802.11 DCF over Correlated Fading Channel in MANET," IEEE, pp. 694-697, 2005

[8] IEEE 802.11b, "Part 11: Wireless LAN Medium Access Control (MAC) and Physical Layer (PHY) Specification: High-Speed Physical Layer Extension in the $2.4 \mathrm{GHz}$ Band," 1999.

[9] IEEE Std 802.11n 2009 "Part 11: Wireless LAN Medium Access Control(MAC) and Physical Layer (PHY) specifications: Enhancements for Higher Throughput," Oct. 2009.

[10] D. Skordoulis, Q. Ni, H. Chen, A. P. Stephens, C. Liu and A.Jamalipour, "IEEE 802.11n MAC Frame Aggregation Mechanisms for Next-Generation High-Throughput WLANs," IEEE Wireless Communications, vol. 15, pp.40-47, Feb.2008.

[11] Giuseppe Bianchi, "Performance Analysis of the IEEE 802.11 Distributed Coordination Function," IEEE Journal on Selected Areas in Communications, Vol. 18, No.3, pp. 535547, March 2000.

[12] F. Zheng and J. Nelson, "Adaptive Design for the Packet Length of IEEE 802.11n Networks," in 2008 IEEE Intern. Conf. Communications, Beijing, May.2008, pp. 24902495.

[13] Y. Lin and V. W. S. Wong, "Frame Aggregation and Optimal Frame Size Adaptation for IEEE 802.11n WLANs," in Proc. IEEE GLOBECOM, San Francisco, CA, Nov. 2006, pp. $1-6$.

[14] Ha Cheol Lee, "A MAC Layer Throughput over Error-Free and Error-Prone Channel in The 802.11a/g-based Mobile LAN," MICC 2009, Dec. 2009

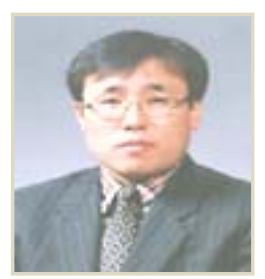

Ha Cheol Lee was born in Korea in 1960. He received B.E., M.E. and Ph. D. from The Department of Information and Communication Engineering, Korea Aerospace University, Korea in 1983, 1990 and 1999 respectively. $\mathrm{He}$ is working as professor in Dept. of Information and Communication Eng. of Yuhan University in Korea since 1995. He was a research engineer in ETRI (Electronic Telecommunication Research Institute) and KT (Korea Telecom) from 1983 to 1995 in Korea. He also acquired certificate of professional engineer in 1993 in Korea. His areas of interest are wireless LAN, Home network and PAN design. 